\title{
Glossary of specific terms and notational symbols
}

\author{
MC Middle Chinese \\ OC Old Chinese \\ * historical reconstruction \\ ** ungrammatical sentence or expression \\ ? $\quad$ unidentified meaning \\ ?? marginal sentence or expression \\ ! pejorative language \\ *() the bracketed element is obligatory \\ () the bracketed element is optional \\ $>$ phonological/morphological tone change \\ $\sim \quad$ phonological free variant of tone \\ [] the bracketed element is phonemic \\ / / the bracketed element is allophonic
}


